\title{
The effect of stellar activity on the $\mathrm{Li}$, 6708, $\mathrm{Na}$, 5896 and $\mathrm{K}, 7699 \AA$ lines
}

\section{A comparison with the Pleiades, field stars and the Sun}

\author{
D. Barrado y Navascués ${ }^{1}$, R. J. García López ${ }^{2,3}$, G. Severino ${ }^{4}$, and M. T. Gomez ${ }^{4}$ \\ 1 Departamento de Física Teórica, C-XI-506, Universidad Autónoma de Madrid, Cantoblanco, 28049 Madrid, \\ Spain \\ 2 Instituto de Astrofísica de Canarias, 38200 La Laguna, Tenerife, Spain \\ e-mail: rgl@ll.iac.es \\ 3 Departamento de Astrofísica, Universidad de La Laguna, Av. Astrofísico Francisco Sánchez s/n, \\ 38071 \\ La Laguna, Tenerife, Spain \\ 4 Osservatorio Astronomico di Capodimonte, Via Moiariello, 16, 80131 Napoli, Italy \\ e-mail: severino@na.astro.it; gomez@na.astro.it
}

Received 9 June 2000 / Accepted 12 March 2001

\begin{abstract}
An analytical model has been developed to empirically study the effects of stellar spots and faculae on the observed equivalent widths of $\mathrm{Li}$ I 6708 , NaI 5896 and KI $7699 \AA$ lines (and abundances in the case of lithium) in late-type stars, taking into account the changes in the observed magnitudes and colors. Solar spectra corresponding to different active regions are used as input data and a range of filling factors are applied to simulate the surfaces of stars with different levels of activity. Detailed comparisons between predicted and observed photometric colors and equivalent widths are made for late-type stars of the Pleiades and the field. The observed dispersions in K I and Li I equivalent widths for Pleiades stars can be partially accounted by the simultaneous effects of activity on colors and the line formation, indicating that the lithium-rotation connection suggested for $\sim 0.7-0.9 M_{\odot}$ Pleiades stars could be due in part to the stellar activity. However, under realistic values for the filling factors, only a small portion of the observed spread could be explained by these effects.
\end{abstract}

Key words. Sun: activity - Sun: faculae, plages - sunspots - stars: abundances - stars: activity - stars: late type

\section{Introduction}

The formation of neutral alkali resonance lines in the solar photosphere is known to be affected by the presence of active regions and spots on the solar surface, because the fractions of neutral alkali atoms in their ground states are very sensitive to the photospheric temperature structure (see e.g. Giampapa 1984; Caccin et al. 1993; Severino et al. 1994, and references therein). This is expected also to be the case for the photospheres of other late-type (Fto early M-type) stars with similar atmospheric characteristics. Different authors have dealt with the comparison of observed and synthetic spectra of Na I D (5890-5896 ^)

\footnotetext{
Send offprint requests to: D. Barrado y Navascués, e-mail: barrado@pollux.ft.uam.es

* Based on observations collected with the GregoryCoudé telescope, operated on the island of Tenerife by the Universitäts-Sternwarte Göttingen in the Spanish Observatorio del Teide of the Instituto de Astrofísica de Canarias.
}

and K I $7699 \AA$ lines in stars of different spectral types (Covino et al. 1993; Andretta et al. 1997; Tripicchio et al. 1997, 1999; Short \& Doyle 1998), showing the benefits and difficulties in using the lines of neutral alkali to probe the effect of activity on the photospheres of these stars.

Both the LiI 6708 and KI $7699 \AA$ features are resonance lines with similar excitation potentials and similar formation conditions: any line formation effect which affects the K I line should also affect the Li I line. In contrast with potassium, lithium is destroyed inside the stellar interior by $(p, \alpha)$ nuclear reactions in those layers where the temperature reaches $\sim 2.510^{6} \mathrm{~K}$. The $\mathrm{K}$ I line appears then as a key tool for identifying and isolating the possible effects of activity on the observed $\mathrm{Li}$ abundances in latetype stars, since its surface abundance does not depend on stellar age, rotational velocity, etc.

Open clusters, due to the common properties shared by the stars belonging to them (same age and metallicity), provide excellent opportunities to study the details 
of stellar structure and evolution. Lithium abundances have been derived for a significant number of clusters and different mixing mechanisms have been invoked to explain the observed patterns (see recent reviews by Balachandran 1995; Pinsonneault 1997; Martín 2001; Vauclair 2001). These observations indicate that the abundance decreases with temperature and stellar age, and that there is a starto-star scatter of the $\mathrm{Li}$ abundance for the same temperature. In particular, the existence of a lithium-rotation connection has been suggested for $\sim 0.7-0.9 M_{\odot}$ stars in young clusters such as the Pleiades (Butler et al. 1987; García López et al. 1991a,b; Soderblom et al. 1993; García López et al. 1994; Jones et al. 1996) and Alpha Persei cluster (Balachandran et al. 1988; Randich et al. 1998), indicating that fast rotators, as a group, show higher lithium abundances than slow rotators. Balachandran et al. (1998) discuss different aspects of the lithium-rotation connection and mixing in late-type stars. Note, however, that slightly older clusters do not clearly show this connection, as in the case of M 35 (Barrado y Navascués et al. 2001b). Alternatively, it has also been suggested that chromospheric activity could affect the formation of the Li line, partially accounting for the observed dispersion (Houdebine \& Doyle 1995; Barrado y Navascués 1996; Russel 1996; Jeffries 1999; King et al. 2000). In this case the Li-rotation connection would be a Li-activity-rotation relation (higher rotation means higher activity) and the observed dispersion in $\mathrm{Li}$ equivalent widths $(W \mathrm{~s})$ would not (or only in part) correspond to a real dispersion in $\mathrm{Li}$ abundances.

Soderblom et al. (1993) found that the KI $7699 \AA$ equivalent widths of K-type stars in the Pleiades exhibited a similar trend to that of $\operatorname{Li}$ I $6708 \AA$ but with a lower spread. They concluded in their study that activity could only partially influence the observed Li spread. Stuik et al. (1997) compared observed and synthetic Li I and K I spectra (affected by the presence of spot and plages) for late-type Pleiades stars. They found that these lines are not sensitive to the presence of a chromosphere, and that they respond to the effects of activity on the stratification in the deep photosphere. However, they could not establish whether or not magnetic activity is the major contributor to the observed K I (and Li I) scatter. More recently, King et al. (2000) addressed this problem by using differential $\mathrm{Li}$ abundances (with respect to fitted values vs. effective temperature) and differential K I equivalent widths. They invoked an incomplete treatment of line formation (in particular, the role of ionization in reducing the the Li I line optical depth; Houdebine \& Doyle 1995) to explain the correlations found between differential $\mathrm{Li}$ and $\mathrm{K}$ values and differences in stellar activity (measured using the Ca II IR triplet).

None of these previous works has clearly established the quantitative effects of stellar activity on the KI and Li I lines. In this paper, we have adopted an empirical approach to this study and included the Na I $5896 \AA$ line as a complementary indicator. An analytical model has been developed to evaluate the simultaneous effect of surface inhomogeneities on the observed line equivalent widths and stellar photometric colors. The inputs to this model come from high-quality, high-resolution observations carried out on different regions of the solar disk. Section 2 briefly describes the observational material, while Sect. 3 provides the details of the model. A comparison between model predictions and photometric data for the Pleiades is shown in Sect. 4. Section 5 discusses the effect of stellar spots and faculae on the alkali and compares these results with K I and Li I measurements in late-type Pleiades stars. Finally, Sect. 6 summarizes our main conclusions.

\section{Observational material}

The observations used in this work were carried out in September 1994, using the echelle spectrograph of the Gregory-Coudé telescope located at the Teide Observatory, Tenerife (Spain). This instrument provides a great stability together with very high spectral resolution. Spectra with a resolving power of $\lambda / \Delta \lambda \sim 3.510^{5}$ were recorded in each spectral range using a $1024 \times 1024$ pixel Thomson CCD $\left(19 \mu \mathrm{m}\right.$ pixel $\left.^{-1}\right)$. Long slit observations were performed in order to obtain a spatial coverage of $160^{\prime \prime}$ in a given measurement. Each final spectrum is typically the average of 20 independent images (50 in the case of $\mathrm{Li}$ ), which were reduced using MIDAS following the usual procedure for solar observations carried out with this instrumentation. Final signal-to-noise ratios are in the range 1000-4000. Additional details on the observations and data reduction can be found in Barrado y Navascués et al. (1995) and Barrado y Navascués (1996). Table 1 shows the equivalent widths measured for different locations of the observed active region and its surroundings. Since our original spectra have very high resolution, we were able to remove either the nearby $\mathrm{TiO}$ and $\mathrm{CN}$ bandswhich appear at low temperatures- in the case of Li I, or the weak lines in the case of K I and Na I. These equivalent widths will be used as the input data for the model proposed in the next section. Additional measurements (for Na and K) were selected from Tripicchio et al. (1997, 1999) for a sunspot at $3700 \mathrm{~K}$.

\section{The model}

We estimate in this section the behavior of the equivalent widths of Na I 5896, Li I 6708 and K I $7699 \AA$ lines associated with the presence of active regions in late-type stars. Stellar spots (and other surface inhomogeneities) affect the observed photometry due to the lower luminosity of the spot in comparison with that of a quiet region of equivalent size. On the other hand, it is known that the equivalent widths $(W \mathrm{~s})$ of the alkali show different values in different solar regions (as can be seen in Table 1) associated with the different temperatures of the line forming regions. A simple mathematical model is used here to compute an "observed" (predicted) color index - in our case, $(B-V)$ - and the corresponding effective temperature, as well as the "observed" (predicted) equivalent widths of 
Table 1. Alkali equivalent widths measured on different solar regions

\begin{tabular}{lccccc}
\hline Line & \multicolumn{5}{c}{ Equivalent width $(\mathrm{m} \AA)$} \\
\cline { 2 - 6 } & Quiet & Faculae & Average Umbra & Central Umbra & Spot 2 \\
\hline Li I 6708 & 3.977 & 3.569 & 20.247 & 49.080 & - \\
K I 7699 & 179.5 & 177.3 & 294.5 & 373.4 & $1139.0^{(1)}$ \\
Na I 5896 & 599.0 & 531.4 & 1118.4 & 1786.6 & $5180.9^{(1)}$ \\
Temperature $(\mathrm{K})$ & 5655 & - & 4870 & 4575 & $3700^{(1)}$ \\
\hline
\end{tabular}

(1) From Brynildsen et al. (private comm.), after Tripicchio et al. (1997, 1999).

the alkali lines for a given set of parameters. This model is intended to reproduce what happens in late-type stars from our knowledge of the solar surface.

The computation starts from a fictitious star free of active regions and having a given effective temperature. Then, different active regions (spots and faculae) are added to its photosphere in a similar fashion to that followed by Pallavicini et al. (1993).

\subsection{Computing the photometry}

Estimating the observed photometry for a stellar disk which contains spots requires the previous computation of the contribution of the region covered by them, i.e. its filling factor, $F_{\text {spot}}$. In a similar way, we should consider the fraction covered by faculae, $F_{\text {faculae, }}$, and the fraction filled with quiet regions. For simplicity, we have supposed that the photometric properties of these two last regions are identical. Therefore, it is only necessary to take into account $F_{\text {spot }}$ and $\left\{1-F_{\text {spot }}\right\}$. We have not considered the effect of limb darkening either.

The absolute magnitude of the region covered by spots can be expressed by:

$$
\begin{aligned}
M_{V, \mathrm{spot}}= & M_{V, \mathrm{star}}^{(B-V)_{\mathrm{spot}}} \\
& -2.5 \log \left\{F_{\mathrm{spot}} \times\left[R_{(B-V)_{\text {quiet }}} / R_{(B-V)_{\mathrm{spot}}}\right]^{2}\right\},
\end{aligned}
$$

where $M_{V \text {,star }}^{(B-V)_{\text {spot }}}$ is the magnitude of a star with color index $(B-V)$ equal to that one of the spot, $R_{(B-V)_{\text {quiet }}}$ is the radius of a star of color equal to the quite region (the real radius of the considered star), and $R_{(B-V)_{\text {spot }}}$ is the radius of a star which would have the same color of the spot.

In the case of the region covered by faculae and the quiet photosphere:

$M_{V, \text { faculae }}=M_{V, \text { star }}^{(B-V)_{\text {faculae }}}-2.5 \log F_{\text {faculae }}$,

and

$M_{V, \text { quiet }}=M_{V, \text { star }}^{(B-V)_{\text {quiet }}}-2.5 \log \left\{1-F_{\text {spot }}-F_{\text {faculae }}\right\}$,

where we have assumed that $M_{V \text {,star }}^{(B-V)_{\text {faculae }}}$ is equal to $M_{V \text {,star }}^{(B-V)_{\text {quiet }}}$. In a similar way it is possible to obtain the absolute magnitude in the $B$ band $-M_{B}$ - for the regions covered by spots, faculae and quiet region, and derive the color indices for each region.

Finally, the observed magnitudes can be predicted using the expression:

$$
\begin{aligned}
M_{V}^{\mathrm{obs}}= & -2.5 \log \left\{10^{-0.4 \times M_{V, \text { spot }}}\right. \\
& \left.+10^{-0.4 \times M_{V, \text { faculae }}}+10^{-0.4 \times M_{V, \text { quiet }}}\right\} .
\end{aligned}
$$

An equivalent expression gives $M_{B}^{\text {obs }}$, allowing the computation of the color index $(B-V)$ obs. The Appendix A contains a detailed justification of these expressions. Some results are shown in Fig. 1.

\subsection{Computing the equivalent widths}

There are several factors which affect the observed equivalent widths. They depend, among other parameters, on the filling factors of spots and faculae. Moreover, the $W$ s depend on the local continuum level. Therefore, it is necessary to compute the ratios $\alpha_{\text {line }}$, between the fluxes from the region covered by spots and the quiet region (and faculae) at the studied wavelengths $-\alpha_{\text {line }}=$ Flux(spot)/Flux(quiet):

$\alpha_{\text {line }}=\frac{\mathrm{e}^{\left(K(\lambda) / T_{\text {quiet }}\right)}-1}{\mathrm{e}^{\left(K(\lambda) / T_{\text {spot }}\right)}-1}$,

where and $K(\lambda)$ is equal to $2.144310^{4}$ for $\mathrm{LiI} 6708$, $2.440810^{4}$ for $\mathrm{NaI} 5896$, and $1.868310^{4}$ for K I 6799 . Then, the equivalent width for a particular line is estimated using the following expression (see Appendix B):

$W_{\text {obs }}^{\text {line }}=\frac{\alpha_{\text {line }} F_{\text {spot }} W_{\text {spot }}^{\text {line }}+F_{\text {faculae }} W_{\text {faculae }}^{\text {line }}+F_{\text {quiet }} W_{\text {quiet }}^{\text {line }}}{\alpha_{\text {line }} \times F_{\text {spot }}+F_{\text {faculae }}+\left[1-F_{\text {spot }}-F_{\text {faculae }}\right]}$,

where $W_{\mathrm{obs}}^{\text {line }}$ is the predicted equivalent width for a certain line and $W_{\text {spot }}^{\text {line }}, W_{\text {faculae }}^{\text {line }}$ and $W_{\text {quiet }}^{\text {line }}$ are the actual equivalent widths of the spot, faculae and quiet region, respectively.

\subsection{Effective temperatures, radii, colors, and magnitudes}

Tripicchio et al. (1999) have shown that the potassium equivalent width at $7699 \AA$ is an excellent temperature 

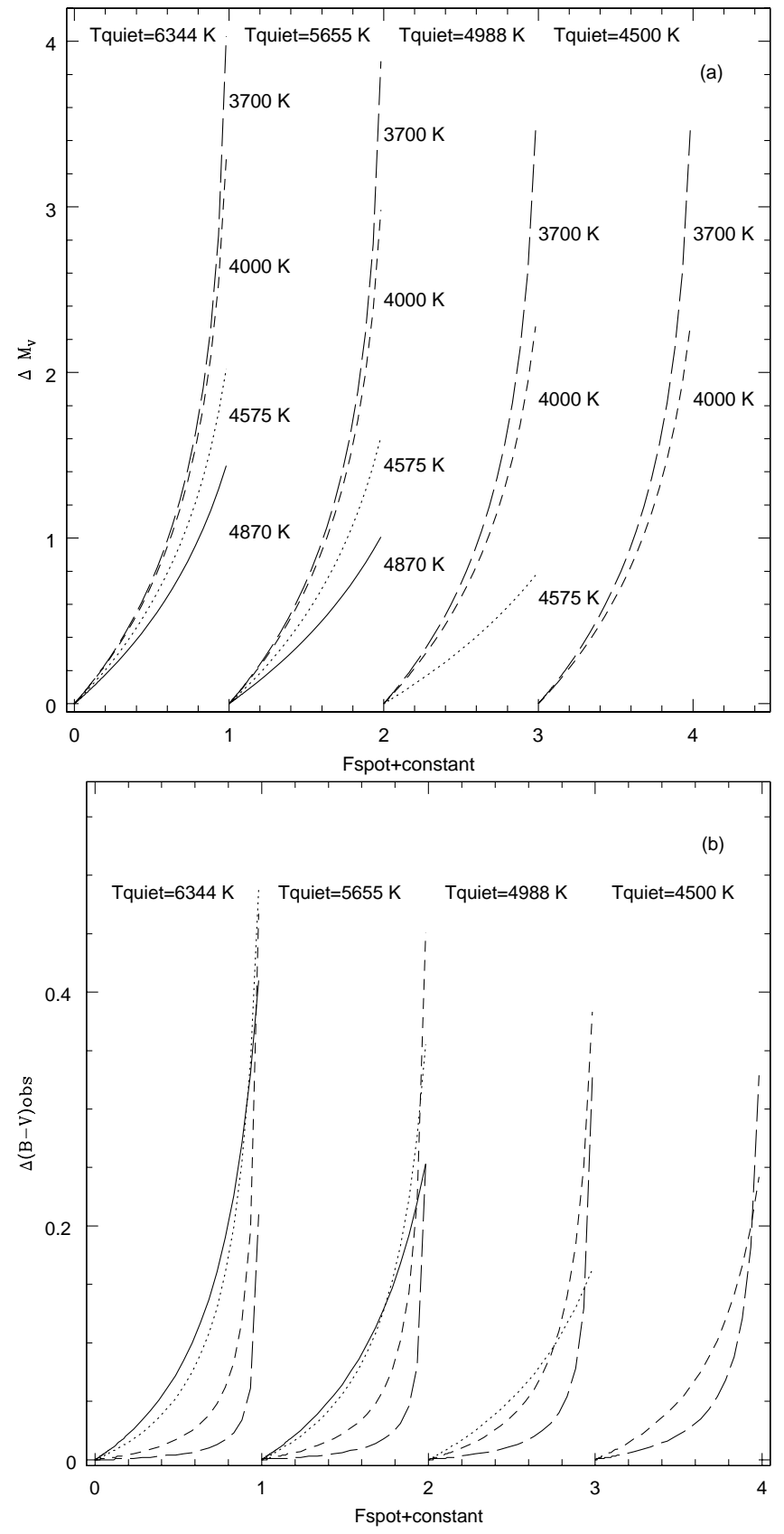

Fig. 1. Results of our model for a star with $T_{\text {quiet }}=6344$, 5655,4988 and $4500 \mathrm{~K}$. The solid line shows the computation for a spot at $4870 \mathrm{~K}$, the dotted line represents the values for a spot at $4575 \mathrm{~K}$, the short dashed line corresponds to $4000 \mathrm{~K}$. Finally, the long dashed line represents the computation for a spot at $3700 \mathrm{~K}$. a) Variation of the visual magnitude, $\Delta M_{V}$, against the filling factor of the spots, $F_{\text {spot }}$. For clarity, we have added one, two and three units to the models having $T_{\text {quiet }}$ equal to 5655,4988 and $4500 \mathrm{~K}$, respectively. b) Dependence of variation of the observed color index $(B-V)$ on the spot filling factor

indicator (in a very well defined spectral range). Therefore, we have computed the effective temperatures from their expression:

$\log (W(\mathrm{~K}) / 7699)=-2.85+3.2910^{-5} \times T_{\text {eff }}-1.4310^{-7}$

$$
\times T_{\text {eff }}^{2}+1.1110^{-11} \times T_{\text {eff }} \text {, }
$$

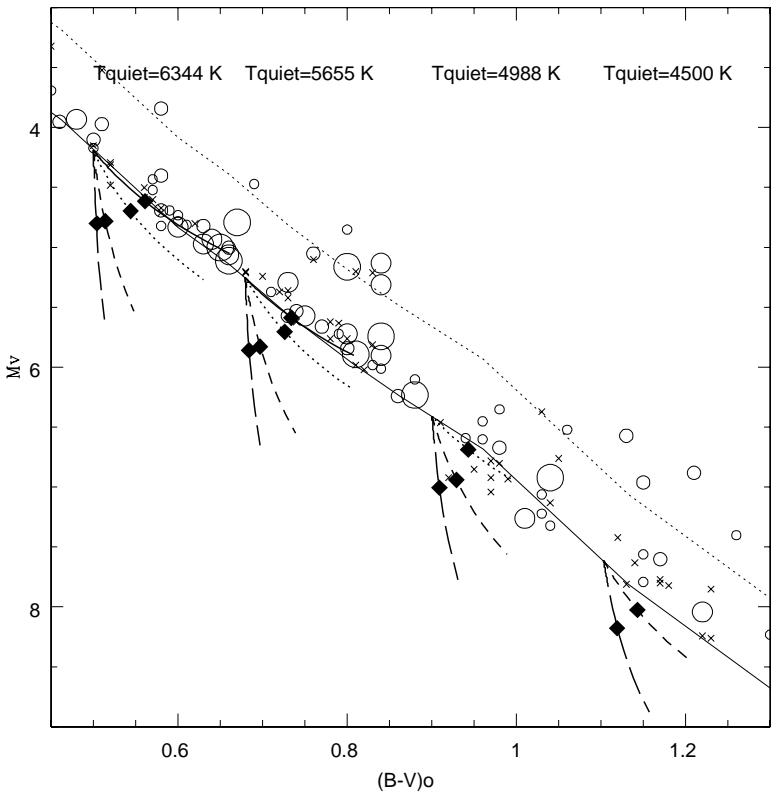

Fig. 2. Visual absolute magnitude against the color index $(B-V)$ for four cases presented in this study (thin-solid, dotted, short dashed and long dashed lines for spots with 4870 , 4575,4000 and $3700 \mathrm{~K}$, respectively). We have computed the observed photometry for stars with $T_{\text {quiet }}=6344,5655,4988$ and $4500 \mathrm{~K}$, corresponding to $(B-V)=0.50,0.68,0.90$ and 1.10, respectively. The MS appears as a thick solid line, whereas the MS for twin stars is represented as a short dashed line $\left(\mathrm{MS}-0.75^{\mathrm{mag}}\right)$. Pleiades data are displayed as open circles and the size increases with increasing stellar activity. Star without known activity appear as plus symbols. Solid diamonds represent our calculations for $F_{\text {spot }}=0.50$. All calculations are displayed up to $F_{\text {spot }}=0.80$

Color indices $(B-V)$ were derived from the Thorburn et al. (1993) scale temperature, namely:

$T_{\text {eff }}=\frac{5040}{0.5247+0.5396 \times(B-V)}$,

Stellar radii were estimated from $(B-V)$ colors, after Schmidt-Kaler (1982).

Absolutes magnitudes were derived using a Pleiades empirical main sequence (MS, see Fig. 2), with a color excess of $E(B-V)=0.04$ and a distance modulus of $(m-M)=5.728$. Other examples of empirical main sequences for the Pleiades, Alpha Persei cluster, M 35 and field stars can be found in Bouvier et al. (1998); Stauffer et al. (1999); Barrado y Navascués et al. (2001b) and Barrado y Navascués et al. (1999), respectively.

\section{The photometry of active solar-type stars}

We have performed a series of simulations under different conditions and obtained final values of "observed" (predicted) photometry for a main sequence star. This star has an effective temperature corresponding to that of the quiet region, and we have assumed different filling factors for the spots (in principle, the photometry is not affected by the presence of faculae). We have used different 
Table 2. Input model parameters for Pleiades and field stars. Columns 4 and 6 provide the $(B-V)$ and radius of a star with the temperature of the spot

\begin{tabular}{|c|c|c|c|c|c|c|c|}
\hline \multirow{2}{*}{$\begin{array}{c}\text { Model } \\
(1)\end{array}$} & \multirow{2}{*}{$\begin{array}{c}M_{V} \\
\text { Quiet } \\
(2)\end{array}$} & \multicolumn{2}{|c|}{$(B-V)_{0}$} & \multicolumn{2}{|c|}{$\operatorname{Radii}\left(R_{\odot}\right)$} & \multicolumn{2}{|c|}{ Temperature (K) } \\
\hline & & $\begin{array}{c}\text { Quiet } \\
(3)\end{array}$ & $\begin{array}{c}\text { Spot } \\
(4)\end{array}$ & $\begin{array}{c}\text { Quiet } \\
(5)\end{array}$ & $\begin{array}{c}\text { Spot } \\
(6)\end{array}$ & $\begin{array}{c}\text { Quiet } \\
(7)\end{array}$ & $\begin{array}{c}\text { Spot } \\
(8)\end{array}$ \\
\hline $1 \mathrm{a}$ & 4.192 & 0.500 & 0.946 & 1.218 & 0.794 & 6344 & 4870 \\
\hline $1 b$ & 4.192 & 0.500 & 1.069 & 1.218 & 0.750 & 6344 & 4575 \\
\hline $1 \mathrm{c}$ & 4.192 & 0.500 & 1.363 & 1.218 & 0.630 & 6344 & 4000 \\
\hline $1 d$ & 4.192 & 0.500 & 1.552 & 1.218 & 0.408 & 6344 & 3700 \\
\hline $2 \mathrm{a}$ & 5.253 & 0.679 & 0.946 & 0.921 & 0.794 & 5655 & 4870 \\
\hline $2 \mathrm{~b}$ & 5.253 & 0.679 & 1.069 & 0.921 & 0.750 & 5655 & 4575 \\
\hline $2 \mathrm{c}$ & 5.253 & 0.679 & 1.363 & 0.921 & 0.630 & 5655 & 4000 \\
\hline $2 \mathrm{~d}$ & 5.253 & 0.679 & 1.552 & 0.921 & 0.408 & 5655 & 3700 \\
\hline $3 a$ & 6.411 & 0.900 & 0.946 & 0.811 & 0.794 & 4988 & 4870 \\
\hline $3 b$ & 6.411 & 0.900 & 1.069 & 0.811 & 0.750 & 4988 & 4575 \\
\hline $3 \mathrm{c}$ & 6.411 & 0.900 & 1.363 & 0.811 & 0.630 & 4988 & 4000 \\
\hline $3 \mathrm{~d}$ & 6.411 & 0.900 & 1.552 & 0.811 & 0.408 & 4988 & 3700 \\
\hline $4 \mathrm{c}$ & 7.612 & 1.103 & 1.363 & 0.737 & 0.630 & 4500 & 4000 \\
\hline $4 d$ & 7.612 & 1.103 & 1.552 & 0.737 & 0.408 & 4500 & 3700 \\
\hline
\end{tabular}

Table 3. Sodium and potassium equivalent widths for Pleiades and field stars, in $\mathrm{m} \AA$

\begin{tabular}{|c|c|c|c|c|c|c|}
\hline \multirow{2}{*}{$\begin{array}{c}\text { Model } \\
(1)\end{array}$} & \multicolumn{2}{|c|}{ Temperature (K) } & \multicolumn{2}{|c|}{$W(\mathrm{Na} I 5896)(\mathrm{m} \AA)$} & \multicolumn{2}{|c|}{$W(\mathrm{~K}$ I7699) $(\mathrm{m} \AA)$} \\
\hline & $\begin{array}{c}\text { Quiet } \\
(2)\end{array}$ & $\begin{array}{c}\text { Spot } \\
(3)\end{array}$ & $\begin{array}{c}\text { Quiet } \\
(4)\end{array}$ & $\begin{array}{l}\text { Spot } \\
(5)\end{array}$ & $\begin{array}{c}\text { Quiet } \\
(6)\end{array}$ & $\begin{array}{l}\text { Spot } \\
(7)\end{array}$ \\
\hline $1 \mathrm{a}$ & 6344 & 4870 & $348.9^{(1)}$ & $1118.4^{(2)}$ & $131.1^{(5)}$ & $294.5^{(2)}$ \\
\hline $1 \mathrm{~b}$ & 6344 & 4575 & $348.9^{(1)}$ & $1786.6^{(2)}$ & $131.1^{(5)}$ & $373.4^{(2)}$ \\
\hline $1 \mathrm{c}$ & 6344 & 4000 & $348.9^{(1)}$ & $3522.9^{(3)}$ & $131.1^{(5)}$ & $595.9^{(5)}$ \\
\hline $1 d$ & 6344 & 3700 & $348.9^{(1)}$ & $5198.9^{(4)}$ & $131.1^{(5)}$ & $1139.0^{(4)}$ \\
\hline $2 \mathrm{a}$ & 5655 & 4870 & $599.0^{(1,2)}$ & $1118.4^{(2)}$ & $179.5^{(2,5)}$ & $294.5^{(2)}$ \\
\hline $2 \mathrm{~b}$ & 5655 & 4575 & $599.0^{(1,2)}$ & $1786.6^{(2)}$ & $179.5^{(2,5)}$ & $373.4^{(2)}$ \\
\hline $2 \mathrm{c}$ & 5655 & 4000 & $599.0^{(1,2)}$ & $3522.9^{(3)}$ & $179.5^{(2,5)}$ & $595.9^{(5)}$ \\
\hline $2 \mathrm{~d}$ & 5655 & 3700 & $599.0^{(1,2)}$ & $5198.9^{(4)}$ & $179.5^{(2,5)}$ & $1139.0^{(4)}$ \\
\hline $3 a$ & 4988 & 4870 & $1167.4^{(1)}$ & $1118.4^{(2)}$ & $264.5^{(5)}$ & $294.5^{(2)}$ \\
\hline $3 b$ & 4988 & 4575 & $1167.4^{(1)}$ & $1786.6^{(2)}$ & $264.5^{(5)}$ & $373.4^{(2)}$ \\
\hline $3 \mathrm{c}$ & 4988 & 4000 & $1167.4^{(1)}$ & $3522.9^{(3)}$ & $264.5^{(5)}$ & $595.9^{(5)}$ \\
\hline $3 \mathrm{~d}$ & 4988 & 3700 & $1167.4^{(1)}$ & $5198.9^{(4)}$ & $264.5^{(5)}$ & $1139.0^{(4)}$ \\
\hline $4 \mathrm{c}$ & 4500 & 4000 & $2154.7^{(1)}$ & $3522.9^{(3)}$ & $377.7^{(5)}$ & $595.9^{(5)}$ \\
\hline $3 \mathrm{~d}$ & 4500 & 3700 & $2154.7^{(1)}$ & $5198.9^{(4)}$ & $377.7^{(5)}$ & $1139.0^{(4)}$ \\
\hline
\end{tabular}

(1) From $W\left(\mathrm{Na}\right.$ I5896) $=0.47310^{(-0.788+1.311(B-V))}$, adapted from Tripicchio et al. (1997).

(2) Measured. See Table 1.

(3) From field stars. See Fig. 3c.

(4) From direct measurement. See Tripicchio et al. (1999).

(5) From $W\left(\right.$ K I7699) $=1.04210^{(1.719+0.762(B-V))}$, adapted from Tripicchio et al. (1999).

spots: one of them corresponds to the average of the actual sunspot. The other one is the central part of it - the nucleus of the umbra -, with lower temperature (4870 and $4575 \mathrm{~K}$, respectively). We have also carried out the computations for spots at 4000 and $3700 \mathrm{~K}$. The model was applied to stars with the following initial temperatures: 6344 , 5655,4988 and $4500 \mathrm{~K}$, corresponding to $(B-V)=0.500$, $0.679,0.900$ and 1.100 , respectively. Table 2 lists the initial model parameters (magnitudes, colors, radii, effective temperatures). The initial values of potassium and sodium equivalent widths adopted for them are shown in Table 3. Lithium equivalent widths and abundances are listed in Tables $4 \mathrm{a}$ and $4 \mathrm{~b}$, in the case of the solar-like case and the Pleiades, respectively.

Figure 1a shows the variation of the visual magnitude $\left(\Delta M_{V}\right.$, defined as the difference between the magnitude 
Table 4. a) Lithium equivalent widths and abundances for a solar-like case

\begin{tabular}{ccccccccc}
\hline Model & \multicolumn{2}{c}{ Temperature $(\mathrm{K})$} & & $A(\mathrm{Li})$ & $W(\mathrm{Li})$ & & $A(\mathrm{Li})$ & $W(\mathrm{Li})$ \\
\cline { 2 - 3 } \cline { 7 - 8 } & Quiet & Spot & & Quiet & Quiet & & Spot & Spot \\
(1) & $(2)$ & $(3)$ & & $(4)$ & $(5)$ & & $(6)$ & $(7)$ \\
\hline 1a & 6344 & 4870 & & 1.0 & $1.010^{(1)}$ & & 0.85 & $20.5^{(2)}$ \\
1b & 6344 & 4575 & & 1.0 & $1.010^{(1)}$ & & 0.87 & $49.1^{(2)}$ \\
1c & 6344 & 4000 & & 1.0 & $1.010^{(1)}$ & & 1.00 & $173.0^{(1)}$ \\
1d & 6344 & 3700 & & 1.0 & $1.010^{(1)}$ & & 1.00 & $249.8^{(3)}$ \\
2a & 5655 & 4870 & & 1.0 & $3.977^{(2)}$ & & 0.85 & $20.5^{(2)}$ \\
2b & 5655 & 4575 & & 1.0 & $3.977^{(2)}$ & & 0.87 & $49.1^{(2)}$ \\
2c & 5655 & 4000 & & 1.0 & $3.977^{(2)}$ & & 1.00 & $173.0^{(1)}$ \\
2d & 5655 & 3700 & & 1.0 & $3.977^{(2)}$ & & 1.00 & $249.8^{(3)}$ \\
3a & 4988 & 4870 & & 1.0 & $20.4^{(1)}$ & & 0.85 & $20.5^{(2)}$ \\
3b & 4988 & 4575 & & 1.0 & $20.4^{(1)}$ & & 0.87 & $49.1^{(2)}$ \\
3c & 4988 & 4000 & & 1.0 & $20.4^{(1)}$ & & 1.00 & $173.0^{(1)}$ \\
3d & 4988 & 3700 & & 1.0 & $20.4^{(1)}$ & & 1.00 & $249.8^{(3)}$ \\
4c & 4500 & 4000 & & 1.0 & $77.0^{(1)}$ & & 1.00 & $173.0^{(1)}$ \\
3d & 4500 & 3700 & & 1.0 & $77.0^{(1)}$ & & 1.00 & $249.8^{(3)}$ \\
\hline
\end{tabular}

(1) From Soderblom et al. (1993) curves of growth, using $A(\mathrm{Li})=1.00$.

(2) Measured. See Table 1.

(3) From Pavlenko et al. (1996) curves of growth, using $A(\mathrm{Li})=1.00$.

of a star only covered by quiet regions and the observed magnitude; always positive) against the filling factor of the spots, $F_{\text {spot }}$. The solid line shows the results for a spot at $4870 \mathrm{~K}$, the dotted line represents the calculation for a spot at $4575 \mathrm{~K}$, whereas the short and long dashed lines represent the values for spots with 4000 and $3700 \mathrm{~K}$, respectively. Although the luminosity can decrease by a factor 10 in the most extreme case, a more realistic situation can be considered in the range $0.0 \leq F_{\text {spot }} \leq 0.5$, which, depending on these particular four spots, corresponds to maximum variations of $\Delta V \sim 0.70-0.60 \mathrm{mag}$. These values have been observed in several active binary systems such as CF Tuc, BD $+25^{\circ} 161$, AY Cet, CC Eri, UX Ari, etc. (e.g., Strassmeier et al. 1993; Strassmeier 1995, and references therein).

Figure 1b displays the dependence of the observed color index $(B-V)$ on the spot filling factor. It is seen that the color can change considerably, a phenomenon which could have important consequences when comparing stars having a high level of activity with inactive stars (i.e., rapid versus slow rotators) at the same observed color, because the color of the quiet photosphere is bluer than the observed color. Note the different behavior for different $T_{\text {quiet }}$, due to the fact that the $V$ filter is centered around $\sim 5500 \AA$, which corresponds to a black-body temperature between the spots at 4870 and $4575 \mathrm{~K}$, whereas the $B$ filter is centered at $\sim 4300 \AA$. Therefore, the relative contributions at the filters $B$ and $V$ are very different depending on the spot and quiet photosphere temperatures. Under realistic conditions, this change can be up to
Table 4. b) Lithium equivalent widths and abundances for Pleiades stars

\begin{tabular}{cccccccc}
\hline Model & \multicolumn{2}{c}{ Temperature $(\mathrm{K})$} & & \multicolumn{2}{c}{$A(\mathrm{Li})$} & & \multicolumn{2}{c}{$W(\mathrm{Li})$} \\
\cline { 2 - 3 } \cline { 7 - 8 } \cline { 7 - 8 } (1) & Quiet & Spot & & Quiet & & Quiet & Spot \\
1a & 6344 & 4870 & & $3.02^{(1)}$ & & 80.0 & $313^{(1)}$ \\
1b & 6344 & 4575 & & $3.02^{(1)}$ & & 80.0 & $383^{(1)}$ \\
1c & 6344 & 4000 & & $3.02^{(1)}$ & & 80.0 & $605^{(1)}$ \\
1d & 6344 & 3700 & & $3.02^{(1)}$ & & 80.0 & $993^{(2)}$ \\
2a & 5655 & 4870 & & $2.80^{(1)}$ & & 140.0 & $284^{(1)}$ \\
2b & 5655 & 4575 & & $2.80^{(1)}$ & & 140.0 & $345^{(1)}$ \\
2c & 5655 & 4000 & & $2.80^{(1)}$ & & 140.0 & $510^{(1)}$ \\
2d & 5655 & 3700 & & $2.80^{(1)}$ & & 140.0 & $632^{(2)}$ \\
3a & 4988 & 4870 & & $1.70^{(1)}$ & & 84.0 & $107^{(1)}$ \\
3b & 4988 & 4575 & & $1.70^{(1)}$ & & 84.0 & $177^{(1)}$ \\
3c & 4988 & 4000 & & $1.70^{(1)}$ & & 84.0 & $276^{(1)}$ \\
3d & 4988 & 3700 & & $1.70^{(1)}$ & & 84.0 & $382^{(2)}$ \\
4c & 4500 & 4000 & & $0.64^{(1)}$ & & 37.0 & $112^{(1)}$ \\
3d & 4500 & 3700 & & $0.64^{(1)}$ & & 37.0 & $157^{(2)}$ \\
\hline
\end{tabular}

(1) From Soderblom et al. (1993) curves of growth, using $W(\mathrm{Li})$ - quiet - from Fig. 6a.

(2) From Pavlenko et al. (1996) curves of growth, using $W(\mathrm{Li})$ - quiet - from Fig. 6a.

$\Delta(B-V) \sim 0.1$. Since there is a relationship between the color indices and the stellar mass, the mass estimated for the more active star would be lower than its actual value in the case of MS stars. Using the presence of photometric variations due to the modulation with phase, as the star rotates, can help to avoid this bias, although the lack of modulation does not guarantee the absence of this bias, since the spots can be distributed in a homogeneus way on the stellar disk, producing null or very small photometric variations. In fact, studies made with tomographic spectroscopy or Doppler imaging (Strassmeier 1995; Vogt \& Hatzes 1995) and theoretical works (Schüssler 1995), show that the spots in very active systems tend to be concentrated in the poles, staying there for a long period of time (time scale of years), and producing minimum photometric variations.

We have performed several comparisons between the observed data and the predictions of this model on the effect of the presence of surface inhomogeneities on the photometry and the equivalent widths. A first check is provided by the Pleiades open cluster by looking at the location of the active stars in the Color-Magnitude diagram (CMD), where the photometric properties of the late-type stars change due to the presence of surface features with different temperatures. Figure 2 shows the visual absolute magnitude against the dereddened color in$\operatorname{dex}(B-V)$ for the four cases presented in this study. The behavior of the observed photometry was computed using a quiet photosphere with four different color indices $(0.500,0.679,0.900$ and 1.100$)$ corresponding to the effective temperatures listed in Table 2 . The main sequence 
appears as a thin solid line, whereas the locus for twin stars (MS- $0.75^{\mathrm{mag}}$ ) is represented as a dotted line parallel to it. Real data for stars belonging to the Pleiades (Soderblom et al. 1993) are included as open circles and the size increases with increasing stellar activity, measured using the CaII IR triplet at $8542 \AA\left(R_{\lambda=8542}\right)$. Those Pleiades stars without measured calcium appear as plus symbols. Solid diamonds in the figure represent model predictions for $F_{\text {spot }}=0.50$. Our model shows that the presence of spots shifts the location of these stars almost in parallel with the MS, except when the difference between the quiet atmosphere and the stellar spot becomes very large. In this last case, when the spots are at temperatures much lower than the photospheric value, large increments of the observed magnitude with respect to the MS at the same observed color could appear, with almost no change in the color. However, the lack of stars in this region suggests that either such differences are not possible (i.e., $T_{\text {quiet }}=6344 \mathrm{~K}$ and $T_{\text {spot }}=3700 \mathrm{~K}$ ) or the filling factor must be very small in this case. Therefore, for a given observed color index, the active stars would tend to be located in the lower part of the MS (the less luminous region), even below the MS of single and inactive stars. In the most extreme case, they could be found below the Zero Age Main Sequence (ZAMS). However, the comparison with the Pleiades shows that this phenomenon is not observed. Several factors can explain this situation: first, the physical size of the cluster can introduce a scatter in the CMD equivalent to several tenths of magnitude, which can hide the effect of the activity on the photometry (i.e. Barrado y Navascués \& Stauffer 1996). On the other hand, an important fraction of the stars are in fact binaries and each component can have a different degree of activity, a case not included in the assumptions made in our simple model. However, the effect of stellar spot on the observed photometry of star suggests that it might be influencing on the Hipparcos problem (i.e., the fact that the locus of very well known, nearby open clusters whose distance have been measured by the Hipparcos satellite. See Pinsonneault et al. 2000; Robichon et al. 2000, and references therein), in the sense that two open clusters of similar ages but very different rotational velocity distributions might have a shift in the locus of their main sequence when compared with each other. In summary, Fig. 2 shows that the photometry obtained using our model is compatible with the observational data and their uncertainties for situations showing not large differences between $T_{\text {quiet }}$ and $T_{\text {spot }}$ and realistic values of $F_{\text {spot }}$.

\section{The effect of stellar spots and faculae on the alkali}

\subsection{Potassium (K I $7699 \AA)$}

The direct comparison between the $W\left(K_{\text {I }} 7699 \AA\right)$ corresponding to different regions of the solar photosphere indicates that the variations due to the presence of spots have to be small, except for large values of the spot filling factor. Figure 3a shows the relative variation of the observed $W(\mathrm{~K})$ against $F_{\text {spot }}$ for the solar-like case. In the most extreme cases, when $F_{\text {spot }} \sim 1$, changes close to a $300 \%$ can be reached. However, the more realistic values shown in the figure indicate changes between $0 \%$ and $50 \%$. In any case, this kind of variation due to spots should be easily observed, as we show below.

Figure 3a includes several sets of curves: the dotted lines represent the results obtained using a spot at $4575 \mathrm{~K}$ and different values of the faculae filling factor. From top to bottom, $F_{\text {faculae }}=0.00,0.25,0.50$ and 0.75 . The solid lines correspond to the computations for $T_{\text {spot }}=4870 \mathrm{~K}$. Other sets, computed with $T_{\text {spot }}=4000 \mathrm{~K}$ and $T_{\text {spot }}=3700 \mathrm{~K}$ are shown as short and long dashed lines, respectively. A detail of the graph appears in the box (we only display the first two cases). Essentially, the values of $F_{\text {faculae }}$ do not affect the observed value of $W(\mathrm{~K})$, as a consequence of the small difference between the measured equivalent widths in the quiet region and the faculae.

In an analogous way to the previous figure, it is possible to compare the variations of the $W(\mathrm{~K})$ with $(B-V)-$ the values that would be observed, in both cases. There is a quasi-linear relation between both quantities. However, a more interesting comparison is made in Fig. 3b, where we have included real data corresponding to the Pleiades cluster (Soderblom et al. 1993). The size of the symbols, as in Fig. 2, increases with increasing stellar activity (measured using $R_{\lambda=8542}$ ). As noted by Soderblom et al. (1993), in the range $0.50 \leq(B-V) \leq 0.80$ there is a clear trend between activity and the potassium equivalent width for a given value of $(B-V)$ : the larger the activity, the larger $W(\mathrm{~K})$. The reproduction of this behavior has been attempted previously by Stuik et al. (1997), using a purely theoretical model under NLTE conditions. However, their models were not able to match the Pleiades observations (see their Fig. 8).

In order to verify if our empirical approximation would be able to match the behavior observed in the Pleiades, we proceeded as described in Sect. 3. We computed the results using four different situations: a star having a color $(B-V)=0.500$, another star having a color corresponding to that one of the quiet region surrounding the sunspot $(B-V)=0.679-$, a third star with $(B-V)=0.900$, and another with $(B-V)=1.100$, the same values used for the simulations shown in Fig. 2. We used spots at four different temperatures (4870, 4575, 4000 and $3700 \mathrm{~K}$ ), indicated with solid, dotted, short dashed and long dashed lines. The solar symbols (dots plus circles) indicate the location of the quiet sun and the data for the solar spots. A comparison with field dwarfs, from Tripicchio et al. (1999) are displayed in Fig. 3c. In this case, field stars are shown as crosses. Unfortunately, information regarding the activity of these stars is available for only a handful of them.

As can be concluded from Figs. $3 \mathrm{~b}$ and $3 \mathrm{c}$, the behavior of $W(\mathrm{~K})$ is well reproduced despite the simplicity of the procedure. It explains why active stars tend to have larger equivalent widths. The model also explains why there is not a sharp distinction between the location of inactive 

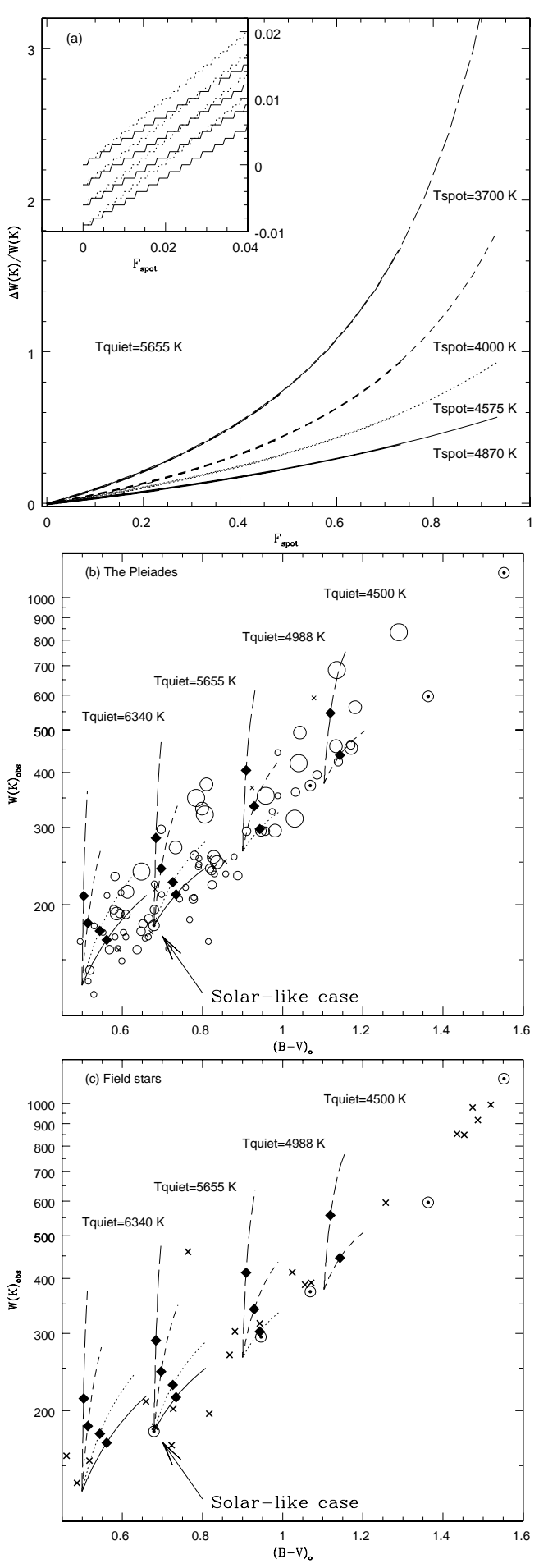

Fig. 3. The effect on potassium. a) Relative variation of the observed potassium equivalent width against the filling factor of the spot. The set displayed with solid lines were computed with $T_{\text {spot }}=4870 \mathrm{~K}$, whereas the set plotted with dotted, short dashed and long dashed lines corresponds to $T_{\text {spot }}=4575 \mathrm{~K}, T_{\text {spot }}=4000 \mathrm{~K}$, and $T_{\text {spot }}=3700 \mathrm{~K}$ For a given set, we computed our results using different faculae filling factors. From top to bottom, $F_{\text {faculae }}=0.00,0.25,0.50$ and 0.75. b) Comparison with Pleiades data. Symbols as in Fig. 2. Values measured/estimated for different sunspots appear with the solar symbol (dot+circle). c) Comparison with field stars (from Tripicchio et al. 1999). These main sequence stars are represented as crosses and active stars: it is predicted that there are locations where it is possible to find stars having high and intermediate activity, or intermediate and low activity. This is because stars having different masses are compared: since the activity of the more massive stars modifies the observed photometry to a greater extent, both stars (the very active and the less active) have similar apparent colors. Note, however, that realistic values of the spot filling factor $\left(F_{\text {spot }} \leq 0.50\right.$; indicated by solid diamonds in Fig. 3b) cannot reproduce those stars having the largest values of the $W$ (K I 7699) for a given color. On the other hand, the figures should be interpreted with some caution, since the model is rather sensitive to the initial conditions (i.e., the initial effective temperature of the spot and the quiet photosphere and the potassium equivalent width of the spot).

\subsection{Sodium ( $\mathrm{Na}$ I $5896 \AA)$}

As it is shown in Table 1 , the $W(\mathrm{Na})$ is much larger in a sunspot than in the quiet region. Therefore, the observed value of the equivalent width changes in a much larger amount than in the case of potassium for the same filling factor of the spot, although the relative variation is similar for both KI and Na I (with respect to their respective equivalent widths). Figures $3 \mathrm{a}$ and $4 \mathrm{a}$ illustrate this fact. In addition, the model predicts that the observed value for $\mathrm{Na}$ I could be smaller than the equivalent width corresponding to the star when it is inactive. This unlikely situation would appear if an important fraction of the surface were covered by active regions (faculae) but having very small spot coverage. In any case, the important result coming from this figure is that it is possible to have a situation in which a high degree of activity corresponds with no variations of the $W(\mathrm{Na})$. This would happen if the active regions were distributed homogeneously, or for certain combinations of the values of $F_{\text {spot }}$ and $F_{\text {faculae }}$.

Figure $4 \mathrm{~b}$ plots predicted $W(\mathrm{Na})$ against $(B-V)$. We have included in the figure, as crosses, data corresponding to field dwarfs (Tripicchio et al. 1997). Note that the field stars could have different abundances of sodium. However, assuming a similar abundance, our model is able, in principle, to explain the scatter of the observed $W(\mathrm{Na})$. Although, to our knowledge, there are no studies about the behavior of this doublet in late-type stars for young clusters, we would expect in principle a similar behavior to the case of K I $7699 \AA$ (i.e., for a given color, the larger the activity, the larger the equivalent width). However, there is a significant difference between Na I 5896 and K I $7699 \AA$ lines: the significant difference of equivalent widths between faculae and quiet regions for $\mathrm{NaI}$, which is not observed for K I. So, the scatter displayed in Fig. 3b (potassium equivalent width against color index) might be different in a similar plot for sodium. Therefore, a sample of coeval stars of different colors might not show a clear relationship between activity and $W(\mathrm{Na})$, since a star can have a large activity and, simultaneously, change 

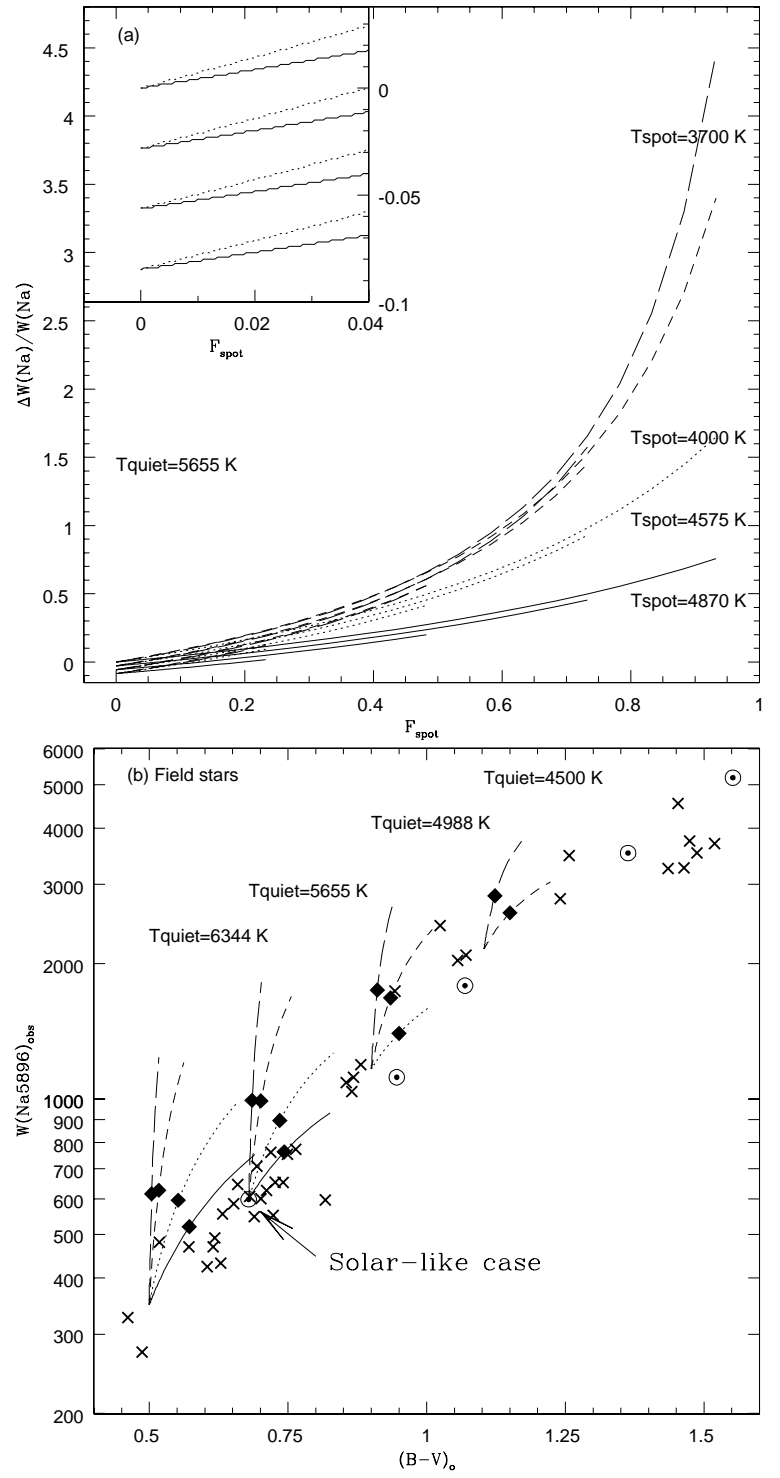

Fig. 4. a) Variations of the sodium equivalent width against the spot filling factor. Symbols as in Fig. 3a. b) Variations of the sodium equivalent width against $(B-V)$ color index. Field dwarfs from Tripicchio et al. (1997) are displayed as crosses

minimally the equivalent width of Na I $5896 \AA$ (if a large fraction of the stellar surface is covered with active regions). This is a very interesting tool to verify the effect of spots and faculae on the equivalent widths of alkali.

\subsection{Lithium (Li। $6708 \AA)$}

\subsubsection{The behavior of the $W(\mathrm{Li})$}

The study of the variation of the lithium equivalent width due to the presence of surface inhomogeneities (spots and faculae) can provide essential hints to understand the abundances derived for stars with a high level of activity, such as chromospherically active binary systems and rapid rotators belonging to young clusters.

Our model predicts a large variation in $W(\mathrm{Li})$, even close to $3500 \%$, due to the presence of spots. This can be
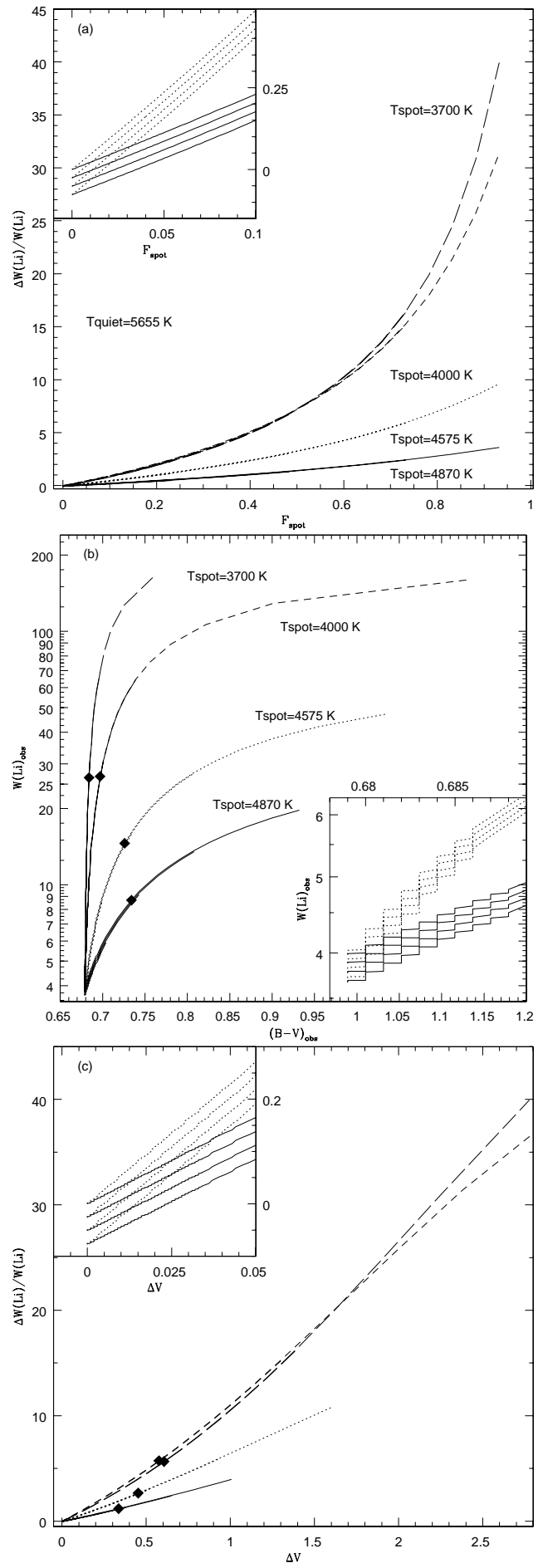

Fig. 5. a) Variations of the lithium doublet equivalent width against the spot filling factor. b) Lithium equivalent width versus $(B-V)$ color index. c) Variations of the lithium doublet equivalent width against photometric variations. A detail of these graphs are displayed in the small boxes. Symbols as in Fig. 3a

clearly seen in Fig. 5a. This kind of variation has already been predicted by Giampapa (1984). The behavior of the lithium line is very sensitive to the temperature of the spot. 
As for Na I $5896 \AA$, the presence of faculae is an important factor that can alter the observed equivalent widths to a significant degree, even reducing it when comparing with the characteristic value of a quiet region. Figure 5a shows that a solar-like star could have null variations of $W(\mathrm{Li})$ under a wide variety of situations, all of them realistic. This occurs when the filling factor of the spots is small and a significant part of the photosphere is covered with faculae.

Figure 5b shows the extraordinary change of the $W(\mathrm{Li})$ as the observed $(B-V)$ color increases in the solar-like case, as a consequence of the presence of active regions. The dependence on the spot temperature is evident: the smaller the temperature, the larger the observed $W(\mathrm{Li})$ and color.

Pallavicini et al. (1993), in a quasi-simultaneous study involving photometry and spectroscopy (measuring Li I 6708 and Ca I $6718 \AA$ ) of 4 active stars, did not observe any change in the equivalent width of the Li line, even when these objects presented important photometric variations (up to $0.10 \mathrm{mag}$ ). Our model provides an explanation for this apparent puzzle. Although the values of $F_{\text {spot }}$ which correspond to $\Delta V=0.10 \mathrm{mag}$ could produce important increments in $W(\mathrm{Li})$, taking into account the faculae in the simulation changes the scenario completely. The region covered by faculae has a very reduced value of the $W(\mathrm{Li})$ and tends to dilute the line produced by the quiet region and by the spot. Moreover, the presence of faculae is not detected in the photometric modulation. It is reasonable to assume that the fraction of the stellar disk covered by faculae has to be at least as large as the fraction covered by spots. The final consequence is that a star having high or moderate activity can experience important photometric variations together with reduced variations in the $\mathrm{Li}$ equivalent width (either positive or negative), as Fig. 5c demonstrates, where we represent the relative variation of the equivalent width of lithium against the photometric variation.

\subsubsection{A second comparison with the Pleiades}

An additional check to our model can be performed by comparing it, again, with Pleiades data. Figure 6 shows actual Li data (Soderblom et al. 1993), where the size of the symbols increases with increasing activity. Simulations with different photospheric temperatures are displayed, computed with spots with four temperatures $(4870,4575$, 4000 and $3700 \mathrm{~K}$; solid, and dotted, short and long dashed lines, respectively). As with potassium, the spread on the observed lithium equivalent widths in the Pleiades can be reproduced (Fig. 6a). However, in the lithium case, only part of the spread could be due to the effect of the stellar inhomogeneities, since our curves, even for very large filling factors, do not cover the whole area where the Pleiades stars are located. However, the simulations can explain the trend with stellar activity (for a given color): active stars have a larger value of $W(\mathrm{Li})$. Therefore, part of the
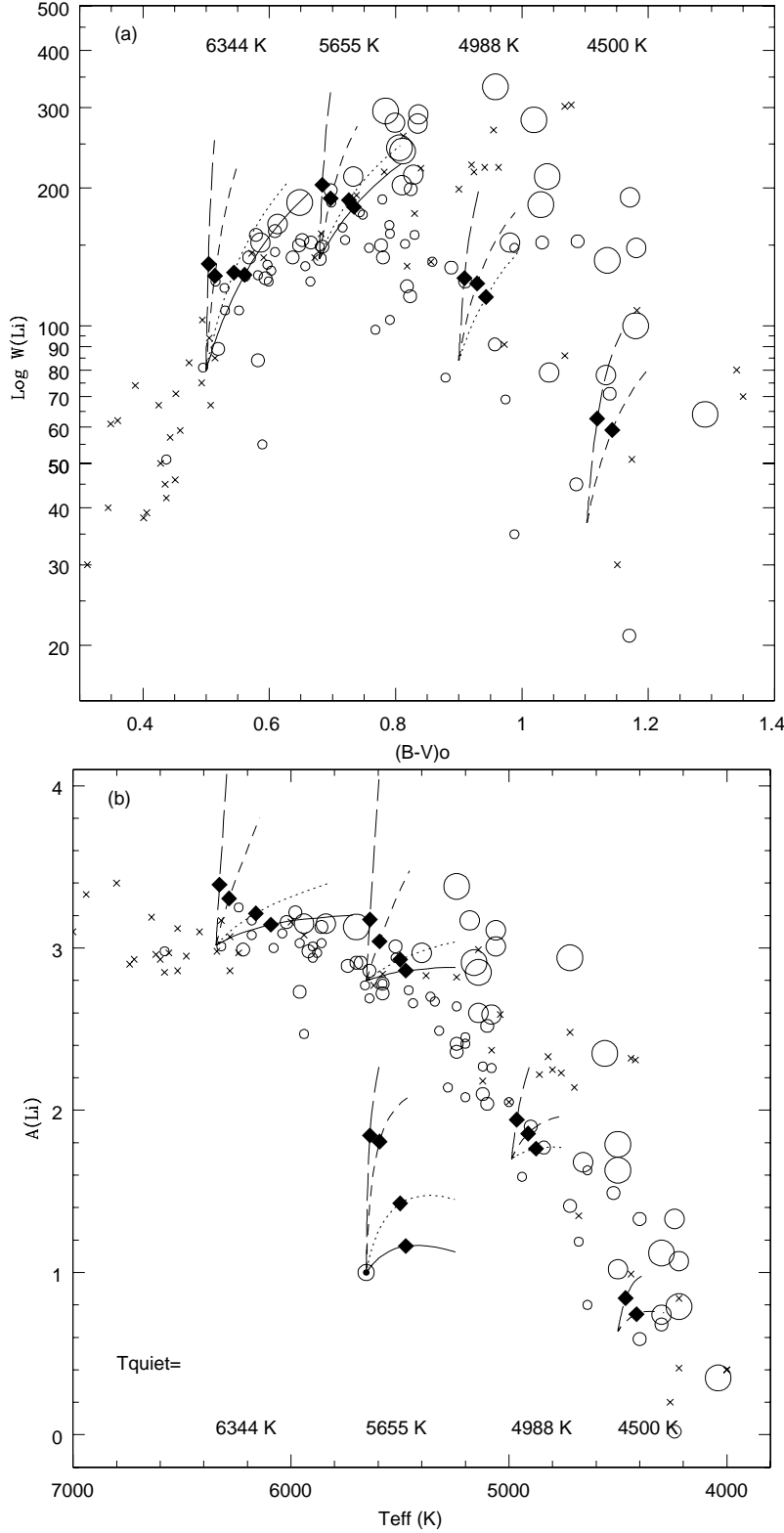

Fig. 6. Comparison with Pleiades data: a) Lithium equivalent width against color. b) Lithium abundance against effective temperature. Symbols as in Fig. 2. We have also included the case for the Sun

spread in the equivalent widths could be due to the simultaneous effect of the surface inhomogeneities on the observed equivalent width and color. Some of the cases would require, however, a (non-realistic) very large filling factor or a large difference between the temperature of the quiet photosphere and the spot.

The $A(\mathrm{Li})-T_{\text {eff }}$ plane (Fig. $6 \mathrm{~b}$, where $A(\mathrm{Li})=$ $\log (\mathrm{Li} / \mathrm{H})+12)$ provides complementary information. Predicted equivalent widths were translated into Li abundances using the curves of growth by Soderblom et al. (1993). In the case of temperatures below $4000 \mathrm{~K}$ (the lowest temperature of these curves of growth), we used those published by Pavlenko et al. (1996). Predicted abundances tend to move the observed values to lower effective 

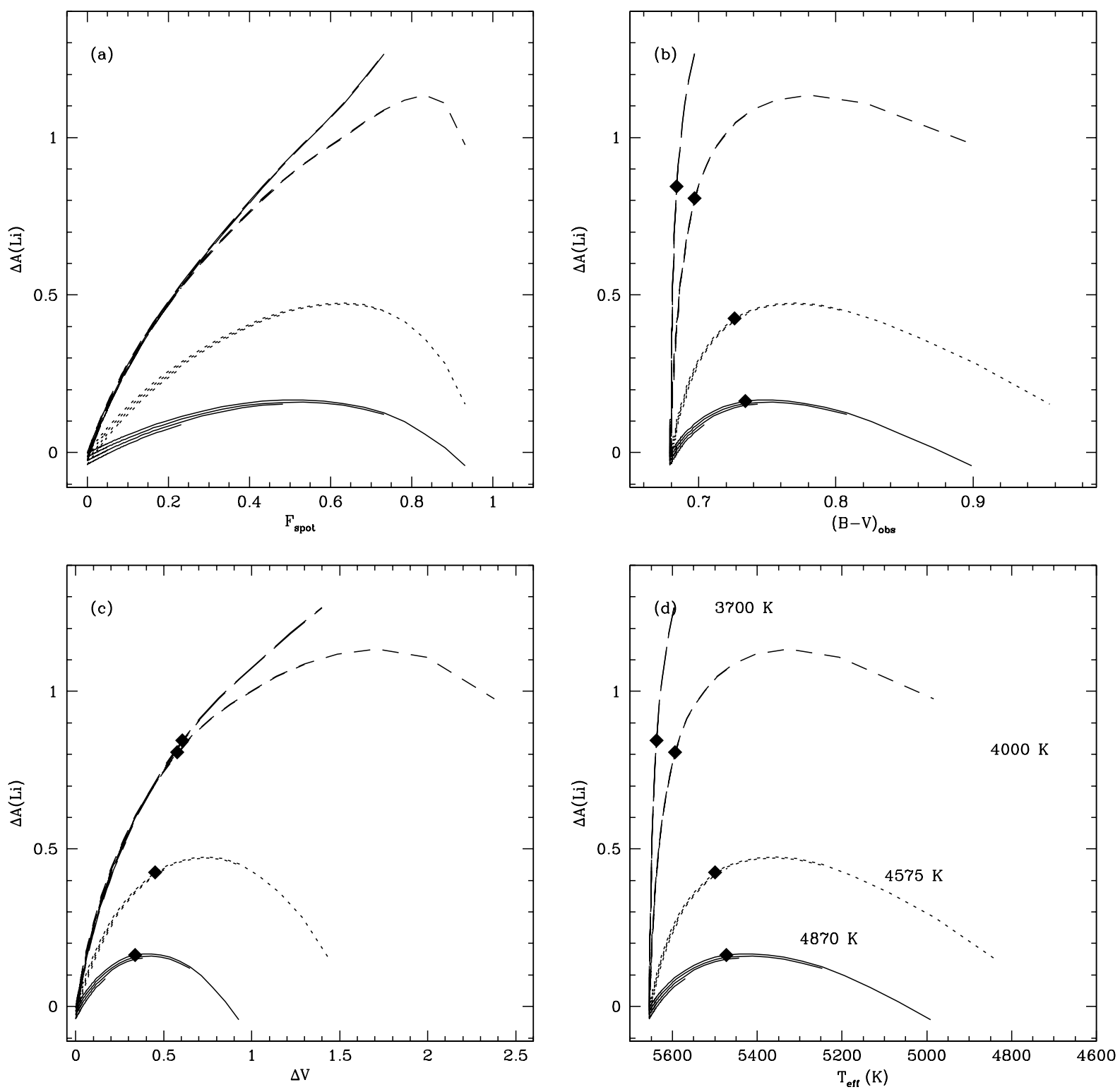

Fig. 7. Behavior of the differences of lithium abundances $\left(A(\mathrm{Li})_{\text {model }}-A(\mathrm{Li})_{\text {initial }}\right)$ against spot filling factor $\left.\mathbf{a}\right),(B-V)$ index b), photometric variations c), and effective temperature d) Symbols as in Fig. 3a. In all cases $A(\mathrm{Li})_{\text {initial }}=1.00$ dex

temperatures for the most active stars, therefore reducing the spread in the abundance and the rotation-activity relation. The lithium abundances for active stars appear then to be correct but their location should be shifted toward higher temperatures in the $A(\mathrm{Li})-T_{\text {eff }}$ plane due to the effect of the spots on the colors. We must note, however, that only very large activity levels would change the morphology of the $T_{\text {eff }}-A(\mathrm{Li})$ plane. Even spot filling factors close to $F_{\text {spot }}=0.50$ move the location of a star in this diagram by $\sim 180 \mathrm{~K}$ only towards cooler temperatures. On the other hand, as discussed before, our simulations do not explain the high lithium abundances in the range 5200$4600 \mathrm{~K}$. Therefore, it seems that these abundances are real and that an important fraction the lithium spread which is present in the figure is real, and that the rotation is a key factor in the evolution of the lithium abundance in the late spectral type stars.

\subsubsection{Uncertainties in the lithium abundance due to the presence of active regions}

We have shown above (see Figs. 5 and 6 ) that the presence of inhomogeneities on the stellar surface could induce important variations in the measured $\mathrm{Li}$ equivalent widths. It is extremely important to establish how these features affect the accuracy of the derived lithium abundances. The presence of spots affects the equivalent width in two different ways: the lithium equivalent width increases, producing a pseudo-abundance larger than the real value. Simultaneously, the color indices are reddened and the lower temperature favors values lower than those corresponding to the quiet photosphere, inducing a reduction in the estimated abundance.

Figure 7a shows the behavior of the abundance provided by the solar model against the spot filling factor for 
a given initial abundance $(A(\mathrm{Li})=1.00$ in this case $)$. $\mathrm{A}$ temperature of $5655 \mathrm{~K}$ for the quiet photosphere, and temperatures of $4870 \mathrm{~K}$ (solid lines), $4575 \mathrm{~K}$ (dotted lines), $4000 \mathrm{~K}$ (short dashed lines), and $3700 \mathrm{~K}$ (long dashed lines) for the spots were used. Different filling factors for the faculae (from top to bottom, 0.00, 0.25, 0.50 and 0.75) were considered. It is clearly seen that the presence of inhomogeneities could introduce important errors in the derived lithium abundance. In this case, they span from -0.05 to +1.2 dex in the worst cases. The decrease in the observed abundance appears when a large fraction of the photosphere is covered by faculae and the filling factor of the spots is small. It is more important when there is a large difference between the spot and quiet photosphere temperatures. These uncertainties are restricted, however, by realistic estimates of the spot filling factor which would yield $\Delta A(\mathrm{Li}) \sim 0.20$ dex.

Figures $7 \mathrm{~b}-\mathrm{d}$ show the behavior of the lithium abundance against the observed color $(B-V)$, the increment in the visual magnitude, and the effective temperature, respectively. These values were determined from $(B-V)$ and the Thorburn et al. (1993) temperature scale. The different observed parameters can change by a considerable amount due to the presence of inhomogeneities. However, the final uncertainties in the derived lithium abundances are limited by the expected values of the filling factors.

Doppler imaging techniques show that active stars have a tendency to concentrate their spots in the poles (Vogt \& Hatzes 1995). Doppler imaging of active Pleiades stars shows that they are, indeed, covered with huge polar spots of presumably long life span (Stout-Batalha \& Vogt 1999). If this controversial fact is true (see Byrne 1995 for a comprehensive discussion about this subject), active stars would show little photometric variations due to inhomogeneities and, simultaneously, the measured equivalent widths would be affected. Therefore, these stars would be several tenths of magnitude fainter than analogous inactive stars (same mass, chemical composition and age), and could show different equivalent widths of the alkali for the same abundances - in the case of lithium, $W(\mathrm{Li})_{\text {measured }}$ could be larger -. However, this situation is not observed when a comparison of the locations of active and inactive stars in Color-Magnitude diagrams or $W(\mathrm{Li})-(B-V)$ plane is performed.

\subsection{Comparison between the alkali}

One of the aims of this work was to compare the behavior of the three alkali elements under several circumstances, establishing the relative variations of the observed equivalent widths in stars with different filling factors of spots and faculae. Figure 8a shows the comparison between potassium and lithium equivalent widths. Pleiades data are included. Symbols are as in Fig. 3b. Active stars tend to be in the top, right-hand side of the diagram. This behavior is qualitatively reproduced with our model.
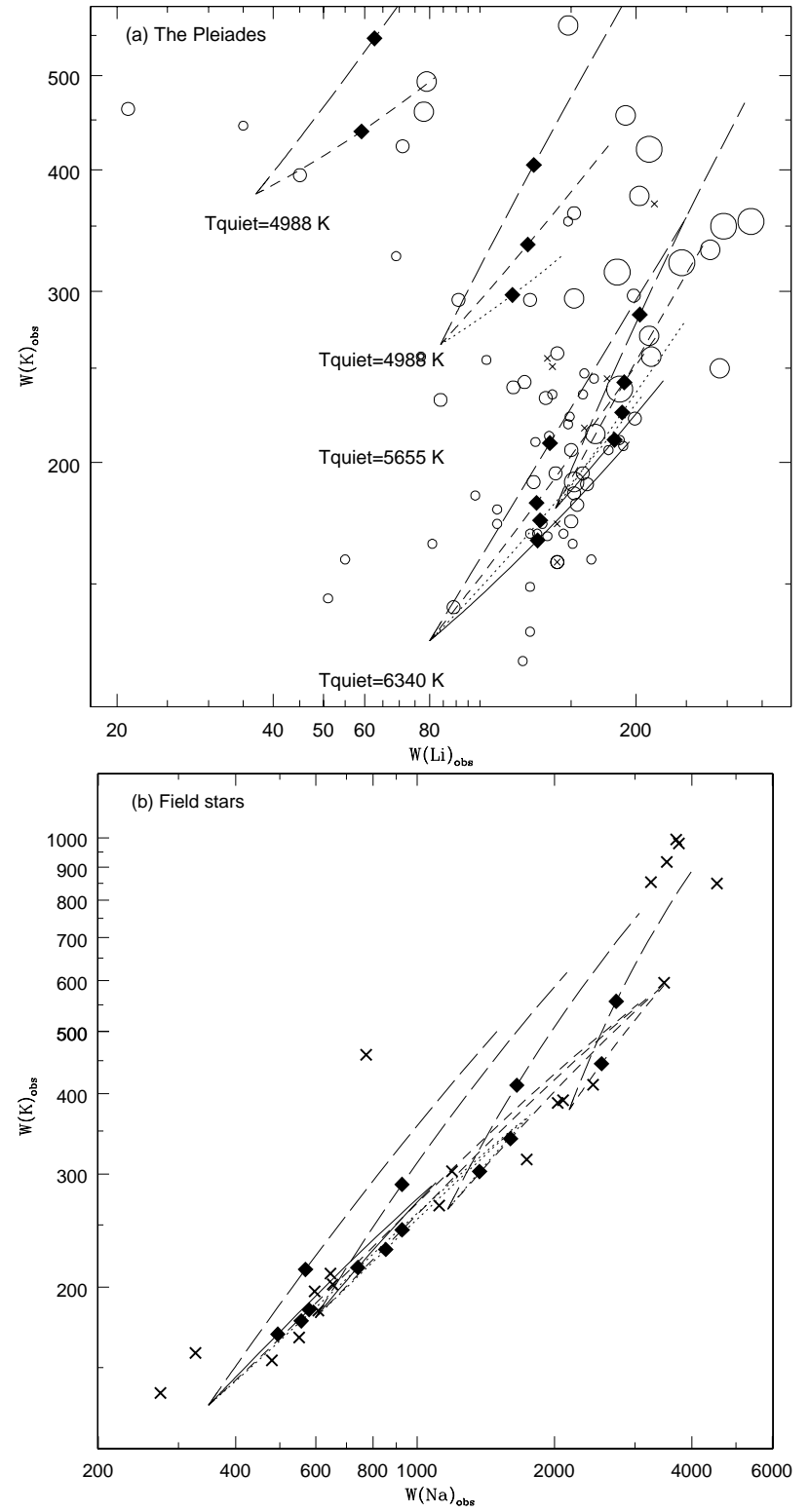

Fig. 8. a) Comparison between the relative variations of potassium and lithium for the Pleiades. Symbols as in Fig. 3b. b) Comparison between the relative variations of potassium and sodium for field stars. Symbols as in Fig. 3c. The last point in our calculations corresponds to $F_{\text {spot }}=0.80$

Figure 8b displays the equivalent widths of sodium and potassium. Data corresponding to field stars are shown as crosses. The dependence of the trend with the spot temperature seems to be very small. It would be very interesting to have similar data for members of a cluster, to determine the effect of the activity on a sample of stars with the same age.

\section{Conclusions}

A simple model has been constructed to study the effects of surface active regions on the behavior of the Li I 6708, Na I 5896, and K I $7699 \AA$ lines in the atmospheres of late-type stars. These effects strongly depend on the 
actual distributions of surface inhomogeneities (spots and faculae in particular) which, unfortunately, are not well known for those objects. They could be distributed in irregular or homogeneous ways and the changes in the alkali line equivalent widths will be affected by the filling factors of the spots and faculae, temperatures of the different regions and the element abundances. Additional observations providing independent estimates of the filling factors, such as direct measurements of the magnetic field via Zeeman splitting (Saar 1995) or empirical relations between the Rossby number and the filling factors (Montesinos \& Jordan 1993), would help to determine the actual values of the colors and equivalent widths and to constrain the input model parameters.

Our simulations predict that active stars can suffer changes in their lithium equivalent widths compared with quiescent objects. However, under normal circumstances (spot filling factors less than 0.5), these variations would not induce significant changes in the derived abundances. A similar conclusion was reached by Pallavicini et al. (1993) by studying the photometry and the Li I $6708 \AA$ line in several stars. Moreover, Pallavicini et al. (1987, 1992), Randich et al. (1993, 1994), Fernández-Figueroa et al. (1993), and Barrado y Navascués et al. (1997, 1998) have studied a large sample of chromospherically active binaries in a time span of several years, and have found no evidences of changes in the lithium equivalent widths that could be related to the phase or to secular changes in the activity.

The comparison between the model predictions and the observations suggests that part of the spread observed in the equivalent widths of $\mathrm{K}$ and $\mathrm{Li}$ lines, as well as in the Li abundances, for the Pleiades could be due to the simultaneous effect of surface inhomogeneities on the observed equivalent widths and photometric colors. At this point it is important to obtain additional information on these lines for other young open clusters where a similar dispersion in Li abundances has been found (eg. Alpha Persei cluster; Randich et al. 1998) to check the model predictions in those cases. Na I D measurements in the Pleiades and other clusters will provide complementary data to witness the behavior of the other two alkali elements.

Acknowledgements. DBN acknowledges the support by the the "Real Colegio Complutense" at Harvard University, the MEC/Fulbright Commission, and "Instituto de Astrofísica de Canarias" and the "Deutsche Forschungsgemeinschaft". This work has been partially supported by the Spanish DGES under projects PB95-1132-C02-01, PB98-0531-C02-02, and "Plan Nacional del Espacio", under grant ESP98-1339-CO2. DBN and RGL appreciate the hospitality of the "Osservatorio Astronomico di Capodimonte". We thank Alfredo Tripicchio for making available his data. We do appreciate the very useful suggestions by the referee, Dr. Sofia Randich.

\section{Appendix A: The photometry}

In order to compute the observed photometry for a star covered with spots and faculae, we have to estimate the individual contribution of each region. The total luminosity can be expressed as:

$L_{V}^{\mathrm{obs}}=L_{V \text {,spot }}+L_{V \text {,faculae }}+L_{V \text {,quiet }}$,

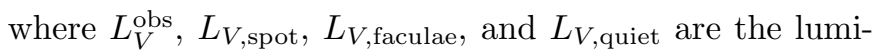
nosities of the observed star, and the contributions from the spots, faculae and quiet region. We have assumed that the photometric properties of the region covered with the faculae are the same as the quiet photosphere. Since:

$L_{V} / L_{V, \odot}=10^{-0.4\left(M_{V}-M_{\odot}\right)}$,

we can conclude that:

$$
\begin{aligned}
M_{V}^{\text {obs }}= & -2.5 \log \left\{10^{-0.4 \times M_{V, \text { spot }}}+10^{-0.4 \times M_{V, \text { faculae }}}\right. \\
& +10^{\left.-0.4 \times M_{V, \text { quiet }}\right\}}
\end{aligned}
$$

where $M_{V \text {,spot }}, M_{V \text {,faculae }}$, and $M_{V \text {,quiet }}$ are the magnitudes of the different regions. We can estimate the first value from:

$M_{V, \text { spot }}=M_{V, \text { star }}^{(B-V)_{\text {spot }}}-2.5 \log \left\{\frac{L_{V, \text { spot }}}{L_{V, \text { star }}^{(B-V)_{\text {spot }}}}\right\}$,

where $M_{V \text {,star }}^{(B-V)_{\text {spot }}}$ is the magnitude of a star at the same $(B-V)$ of the spot and $L_{V, \text { star }}^{(B-V)_{\text {spot }}}$ its luminosity. The ratio between the two luminosities can be re-written as:

$\frac{L_{V, \text { spot }}}{L_{V, \text { star }}^{(B-V)_{\mathrm{spot}}}}=\frac{S_{\mathrm{spot}} \times f_{V, \mathrm{spot}}}{4 \Pi R_{(B-V)_{\mathrm{spot}}}^{2} \times f_{V, \mathrm{star}}^{(B-V)_{\mathrm{spot}}}}$,

where $S_{\text {spot }}$ is the surface covered with spots and $f_{V \text {,spot }}$ is the surface flux of the same region, $R_{(B-V)_{\text {spot }}}$ is the radius of a star with the same color as the spot and $f_{V \text { star }}^{(B-V)_{\text {spot }}}$ is its surface flux. By definition, we have that $f_{V \text {,spot }}=$ $f_{V, \text { star }}^{(B-V)_{\text {spot }}}$. Defining the filling factor as:

$F_{\text {spot }}=\frac{S_{\text {spot }}}{4 \Pi R_{\text {quiet }}^{2}}$,

we have:

$\frac{L_{V, \text { spot }}}{L_{V, \text { star }}^{(B-V)}}=\left[\frac{R_{\text {quiet }}}{R_{(B-V)_{\text {spot }}}}\right]^{2} \times F_{\text {spot }}$.

And we have the magnitude of the region covered with spots:

$$
\begin{aligned}
M_{V, \text { spot }}= & M_{V, \text { star }}^{(B-V)_{\text {spot }}}-2.5 \log \left\{F_{\text {spot }}\right. \\
& \left.\times\left(R_{(B-V)_{\text {quiet }}} / R_{(B-V)_{\text {spot }}}\right)^{2}\right\} .
\end{aligned}
$$

The case of the quiet region and the faculae is simpler, since the correction factor depending on the radii does not exist. Therefore:

$M_{V, \text { faculae }}=M_{V, \text { star }}^{(B-V)_{\text {faculae }}}-2.5 \log F_{\text {faculae }}$

$M_{V, \text { quiet }}=M_{V, \text { star }}^{(B-V)_{\text {quiet }}}-2.5 \log \left\{1-F_{\text {spot }}-F_{\text {faculae }}\right\}$. 


\section{Appendix B: The equivalent width}

Given a star with a quiet region and faculae and spots on its surface, the observed equivalent width contains contributions from each region. The equivalent width is defined as:

$W_{\text {obs }}^{\text {line }}=\int \frac{f(\lambda)^{\text {cont }}-f\left(\lambda_{\mathrm{l}}\right)^{\text {line }}}{f(\lambda)^{\text {cont }}} \mathrm{d} \lambda$,

where $f(\lambda)^{\text {cont }}$ and $f(\lambda)^{\text {line }}$ are the continuum and line fluxes. Since:

$f(\lambda)^{\text {cont }}=F_{\text {spot }} \times f_{\text {spot }}^{\text {cont }}+F_{\text {faculae }} \times f_{\text {faculae }}^{\text {cont }}+F_{\text {quiet }} \times f_{\text {quiet }}^{\text {cont }}$

$f(\lambda)^{\text {line }}=F_{\text {spot }} \times f_{\text {spot }}^{\text {line }}+F_{\text {faculae }} \times f_{\text {faculae }}^{\text {line }}+F_{\text {quiet }} \times f_{\text {quiet }}^{\text {line }}$.

We have that the equivalent width can be re-written as: $W_{\text {obs }}^{\text {line }}=$

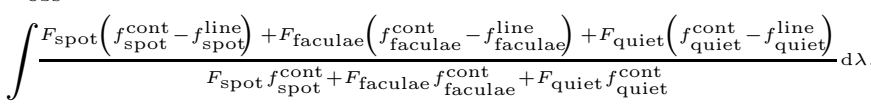

This last equation has three different components, which can be evaluated independently. Therefore, we have $W_{\text {obs }}^{\text {line }}=[1]+[2]+[3]$.

$[1]=\int \frac{F_{\text {spot }}\left(f_{\text {spot }}^{\text {cont }}-F_{\text {spot }}^{\text {line }}\right) / f_{\text {spot }}^{\text {cont }}}{F_{\text {spot }}+F_{\text {faculae }} f_{\text {faculae }}^{\text {cont }} / f_{\text {spot }}^{\text {cont }}+F_{\text {quiet }} f_{\text {quiet }}^{\text {cont }} / f_{\text {spot }}^{\text {cont }}} \mathrm{d} \lambda$.

We can define $\alpha_{\text {line }}=f_{\text {spot }}^{\text {cont }} / f_{\text {quiet } / \text { faculae, }}^{\text {cont }}$ which represents the ratio between continuum fluxes from the spot and the quiet photosphere. Then:

$[1]=\frac{\alpha_{\text {line }} F_{\text {spot }}}{\alpha_{\text {line }} F_{\text {spot }}+F_{\text {faculae }}+F_{\text {quiet }}} \times W_{\text {spot }}^{\text {line }}$.

On the other hand:

$[2]=\int \frac{F_{\text {faculae }}\left(f_{\text {faculae }}^{\text {cont }}-f_{\text {faculae }}^{\text {line }}\right) / f_{\text {faculae }}^{\text {cont }}}{\left(F_{\text {spot }} f_{\text {spot }}^{\text {cont }}+F_{\text {faculae }} f_{\text {faculae }}^{\text {cont }}+F_{\text {quiet }} f_{\text {quiet }}^{\text {cont }}\right) / f_{\text {faculae }}^{\text {cont }}} \mathrm{d} \lambda$.

So

$[2]=\frac{F_{\text {faculae }}}{\alpha_{\text {line }} F_{\text {spot }}+F_{\text {faculae }}+F_{\text {quiet }}} \times W_{\text {faculae }}^{\text {line }}$.

Finally:

$[3]=\int \frac{F_{\text {quiet }}\left(f_{\text {quiet }}^{\text {cont }}-f_{\text {quiet }}^{\text {line }}\right) / f_{\text {faculae }}^{\text {cont }}}{\left(F_{\text {spot }} f_{\text {spot }}^{\text {cont }}+F_{\text {faculae }} f_{\text {faculae }}^{\text {cont }}+F_{\text {quiet }} f_{\text {quiet }}^{\text {cont }}\right) / f_{\text {faculae }}^{\text {cont }}} \mathrm{d} \lambda$.

Which can be written as:

$[3]=\frac{F_{\text {quiet }}}{\alpha_{\text {line }} F_{\text {spot }}+F_{\text {faculae }}+F_{\text {quiet }}} \times W_{\text {quiet }}^{\text {line }}$.

Now, we can evaluate the observed equivalent width:

$W_{\text {line }}^{\text {obs }}=\frac{\alpha_{\text {line }} F_{\text {spot }} W_{\text {spot }}^{\text {line }}+F_{\text {faculae }} W_{\text {faculae }}^{\text {line }}+F_{\text {quiet }} W_{\text {quiet }}^{\text {line }}}{\alpha_{\text {line }} \times F_{\text {spot }}+F_{\text {faculae }}+\left[1-F_{\text {spot }}-F_{\text {faculae }}\right]}$.

\section{References}

Andretta, V., Doyle, J. C., \& Byrne, P. B. 1997, A\&A, 322, 266

Balachandran, S. 1995, ApJ, 446, 203

Balachandran, S., García López, R. J., Kraft, R. P., et al. 1998, in 10th Cambridge Workshop on Cool Stars, Stellar Systems, and the Sun, ed. R. A. Donahue, \& J. A. Bookbinder, ASP Conf. Ser., 154, 111

Balachandran, S., Lambert, D. L., \& Stauffer, J. R. 1988, ApJ, 333,267

Barrado y Navascués, D., De Castro, E., García López, R. J., Sánchez Almeida, J., \& Montesinos, B. 1995, in Stellar Surface Structure, IAU Symp. 176: Poster Proceedings, ed. K. G. Strassmeier (Vienna: Institut für Astronomie der Universität Wien), 123

Barrado y Navascués, D. 1996, Ph.D. Thesis, Universidad Complutense de Madrid

Barrado y Navascués, D., \& Stauffer, J. R. 1996, A\&A, 310, 879

Barrado y Navascués, D., Fernández-Figueroa, M. J., García López, R. J., de Castro, E. \& Cornide, M. 1997, A\&A, 326, 780

Barrado y Navascués, D., de Castro, E., Fernández-Figueroa, M. J., Cornide, M., \& García López, R. J. 1998, A\&A, 337, 739

Barrado y Navascués, D., Stauffer, J. R., Song, I., \& Caillault, J.-P. 1999, ApJL, 520, 123

Barrado y Navascués, D., Stauffer, J. R., Bouvier, J., \& Martín, E. L. 2001a, ApJ, 546, 1006

Barrado y Navascués, D., Deliyannis, C. P., \& Stauffer, J. R. 2001b, ApJ, 549, 452

Bouvier, J., Stauffer, R., Martín, E. L., et al. 1998, A\&A, 336, 490

Butler, R. P., Marcy, G. W., Cohen, R. D., \& Duncan, D. K. 1987, ApJ, 319, L19

Byrne, B. P. 1995, in Stellar Surface Structure, IAU Symp. 176, ed. K. G. Strassmeier, \& J. L. Linsky (Kluwer Academic Publishers), 299

Caccin, B., Gomez, M. T., \& Severino, G. 1993, A\&A, 276, 219

Covino, E., Gomez, M. T., Severino, G., \& Franchini, M. 1993, PASPC, 40, 190

Fernández-Figueroa, M. J., Barrado, D., De Castro, E., \& Cornide, M. 1993, A\&A, 274, 373

García López, R. J., Rebolo, R., Beckman, J. E., \& Magazzù, A. 1991a, in The Sun and Cool Stars: activity, magnetism, dynamos, ed. I. Tuominen, D. Moss, \& G. Rüdiger, Lecture Note in Physics, vol. 380 (Berlin: Springer), 443

García López, R. J., Rebolo, R., Magazzù, A., \& Beckman, J. E. 1991b, MSAIt 62, 187

García López, R. J., Rebolo, R., \& Martín, E. L. 1994, A\&A, 282,518

Giampapa, M. S. 1984, ApJ, 277, 235

Houdebine, E. R., \& Doyle, J. G. 1995, A\&A, 302, 861

Jeffries, R. D. 1999, MNRAS, 309, 189

Jones, B. F., Shetrone, M., Fisher, D., \& Soderblom, D. R. 1996, AJ, 112, 593

King, J. R., Krishnamurthi, A., \& Pinsonneault, M. H. 2000, AJ, 119, 859

Martín, E. L. 2001, in 11th Cambridge workshop on Cool Stars, Stellar Systems and the Sun, ASP Conf. Ser., 223, 207

Montesinos, B., \& Jordan, C. 1993, MNRAS, 264, 900

Pallavicini, R., Cerruti-Sola, M., \& Duncan, D. K. 1987, A\&A, 174,116 
Pallavicini, R., Randich, S., \& Giampapa, M. S. 1992, A\&A, 253,185

Pavlenko, Ya. V., \& Magazzu, A. 1996, A\&A, 311, 961

Pallavicini, R., Cutispoto, G., Randich, S., \& Gratton, R. 1993, A\&A, 267, 145

Pinsonneault, M. H. 1997, ARA\&A, 35, 557

Pinsonneault, M. H., Terndrup, D. M., \& Yuan, Y. 2000, in Stellar Clusters and Associations: Convection, Rotation, and Dynamos, Proceedings from ASP Conf. 198, ed. R. Pallavicini, G. Micela, \& S. Sciortino, 95

Randich. S., Gratton, R., \& Pallavicini, R. 1993, A\&A, 273, 194

Randich, S., Gratton, R., \& Pallavicini, R. 1994, A\&A, 283, 893

Randich, S., Martín, E. L., García López, R. J., \& Pallavicini, R. 1998, A\&A, 333, 591

Robinson, R. D., Thompson, K., \& Innis, J. L. 1986, Proc. Astron. Soc. Australia, 6, 4

Robichon, N. 2000, in Stellar Clusters and Associations: Convection, Rotation, and Dynamos, Proceedings from ASP Conf. 198, ed. R. Pallavicini, G. Micela, \& S. Sciortino, 141

Russel, S. C. 1996, ApJ, 463, 593

Saar, S. H. 1995, in Stellar Surface Structure, IAU Symp. 176, ed. K. G. Strassmeier, \& J. L. Linsky (Kluwer Academic Publishers), 237

Schüssler, M. 1995, in Stellar Surface Structure, IAU Symp. 176, ed. K. G. Strassmeier, \& J. L. Linsky (Kluwer
Academic Publishers), 269

Severino, G., Gomez, M. T., \& Caccin, B. 1994, Solar Surface Magnetism, NATO ASI Ser., C 433, 169

Short, C. I., \& Doyle, J. G. 1998, A\&A, 336, 613

Soderblom, D. R., Jones, B. F., Balachandran, S., et al. 1993, AJ, 106, 1059

Stauffer, J. R., Barrado y Navascués, D., Bouvier, J., et al. 1999, ApJ, 527, 219

Stout-Batalha, N. M., \& Vogt, S. S. 1999, ApJS, 123, 251

Strassmeier, K. 1995, in Stellar Surface Structure, IAU Symp. 176, ed. K. G. Strassmeier, \& J. L. Linsky (Kluwer Academic Publishers)

Strassmeier, K. G., Hall, D. S., Fekel, F. C., \& Scheck, M. 1993, A\&AS, 100, 173

Stuik, R., Bruls, J. H. M. J., \& Rutten, R. J. 1997, A\&A, 322, 911

Thorburn, J. S., Hobbs, L. M., Deliyannis, C. P., \& Pinsonneault, M. H. 1993, ApJ, 415, 150

Tripicchio, A., Gomez, M. T., Severni, G., et al. 1999, A\&A, 345,915

Tripicchio, A., Severino, G., Covino, E., Terranegra, L., \& García López, R. J. 1997, A\&A, 327, 681

Vauclair, S. 2001, in 11th Cambridge worshop on Cool Stars, Stellar Systems and the Sun, ASP Conf. Ser., 223, 227

Vogt, S. S., \& Hatzes, A. P. 1995, in Stellar Surface Structure, IAU Symp. 176, ed. K. G. Strassmeier, \& J. L. Linsky (Kluwer Academic Publishers), 245 